\title{
Numerical methods for plasma physics in collisional regimes
}

 \\ ${ }^{1}$ Department of Mathematics and Computer Science, University of Ferrara, via Machiavelli 35, 44121, \\ Italy \\ ${ }^{2}$ Department of Computing and Mathematical Sciences (CMS), The Annenberg Center, California \\ Institute of Technology, 1200 E. California Blvd., Pasadena, CA 91125, USA \\ ${ }^{3}$ Department of Mathematics, University of California and Los Angeles, Math Sciences Building 520 \\ Portola Plaza Los Angeles, CA 90095, USA
}

(Received 20 June 2014; revised 27 July 2014; accepted 18 August 2014; first published online 10 October 2014)

We consider the development of accurate and efficient numerical methods for the solution of the Vlasov-Landau equation describing a collisional plasma. The methods combine a Lagrangian approach for the Vlasov solver with a fast spectral method for the solution of the Landau operator. To this goal, new modified spectral methods for the Landau integral which are capable to capture correctly the Maxwellian steady state are introduced. A particular care is devoted to the construction of ImplicitExplicit and Exponential Runge-Kutta methods that permit to achieve high-order and efficient time integration of the collisional step. Several numerical tests are reported which show the high accuracy of the numerical schemes here presented.

\section{Introduction}

Coulomb collisions between charged particles in a plasma play a relevant rule in several applications ranging from laser and particle beam interactions with plasma (Pitale 1978; Ghanshyam and Tripathi 1993) to super-thermal radiation (Khabibrakhmanov and Khazanov 2000) and ion transport in fusion reactors (Sydora et al. 2006).

The Landau or Landau-Fokker-Planck equation is a common kinetic model used to describe long-range Coulombian interactions in a weakly ionized gas and is characterized by a nonlinear partial integro-differential equation of the form

$$
\frac{\partial f}{\partial t}+v \cdot \nabla_{x} f+E(t, x) \cdot \nabla_{v} f=\frac{1}{\varepsilon} Q(f, f), \quad v \in \mathbb{R}^{3}, x \in \Omega \subset \mathbb{R}^{3},
$$

where the unknown distribution function $f(t, x, v)$ depends on time $t$, position $x$ and velocity $v$ of particles. In the above equation, $\varepsilon$ is the Knudsen number, $E(t, x)$ the electric field given by the solution of a normalized Poisson equation

$$
E(t, x)=-\nabla_{x} \phi(t, x), \quad \Delta_{x} \phi(t, x)=1-\int_{\mathbb{R}^{3}} f(t, x, v) d v,
$$

and $Q(f, f)$ is the Landau collision operator

$$
Q(f, f)(v)=\nabla_{v} \cdot \int_{\mathbb{R}^{3}} \Phi\left(v-v^{*}\right)\left[\nabla_{v} f(v) f\left(v^{*}\right)-\nabla_{v^{*}} f\left(v^{*}\right) f(v)\right] d v^{*} .
$$

$\dagger$ Email address for correspondence: giacomo.dimarco@unife.it 
In (1.3), the dependence from $(x, t)$ has been omitted for simplicity and $\Phi$ is a $3 \times 3$ non-negative and symmetric matrix that depends on the particles interaction

$$
\Phi(v)=|v|^{\gamma+2} S(v), \quad \gamma \in \mathbb{R} \quad \text { and } \quad S(v)=I-\frac{v \otimes v}{|v|^{2}},
$$

where $I$ is the three-dimensional identity matrix.

Different values of $\gamma$ lead to the classification in hard potentials $\gamma>0$, Maxwellian molecules $\gamma=0$, or soft potentials $\gamma<0$. The latter case involves the Coulombian case $\gamma=-3$, which is of primary importance in plasma physics.

The structure of the Landau operator is similar to the classical Boltzmann collision integral of rarefied gas dynamics (Cercignani 1988) and by standard arguments of kinetic theory one recovers the same physical properties such as the conservation of mass, momentum and energy

$$
\int_{\mathbb{R}^{3}} Q(f, f)(v)\left(\begin{array}{c}
1 \\
v \\
|v|^{2}
\end{array}\right) d v=0
$$

and the entropy production

$$
\frac{d S(t)}{d t}=-\frac{d}{d t} \int_{\mathbb{R}^{3}} f(t, v) \ln (f(t, v)) d v \geqslant 0 .
$$

This implies that the equilibrium states of the Landau operator, i.e. the functions satisfying $Q(f, f)=0$, are given by local Maxwellians

$$
M(v)=\frac{\rho}{\left(2 \pi k_{B} T\right)^{3 / 2}} \exp \left(-\frac{|v-u|^{2}}{2 k_{B} T}\right),
$$

where $k_{B}$ is the Boltzmann's constant, $\rho$ the total mass, $u$ the mean velocity and $T$ the temperature of the plasma given by

$$
\rho=\int_{\mathbb{R}^{3}} f(v) d v, \quad u=\frac{1}{\rho} \int_{\mathbb{R}^{3}} f(v) v d v, \quad T=\frac{1}{3 \rho} \int_{\mathbb{R}^{3}} f(v)(u-v)^{2} d v .
$$

The Landau collision operator (1.3) is obtained as an approximation of the Boltzmann collision operator for Coulomb interactions. In Coulomb collisions, small angle collisions play a more important role than collision resulting in large velocity changes. In such case, the Boltzmann collision operator has no meaning (due to the divergence of the integral even for smooth functions) and a cut-off angular approximation is used to derive the Landau equation in the so-called grazing collision limit (Landau 1936). Several rigorous mathematical derivation of the Landau equation have been performed, we mention here the works of Arsenév (1989), Degond and Lucquin-Desreux (1992), Desvillettes (1992), Rosenbluth et al. (1957) and Alexandre and Villani (2004). For a review of the main mathematical aspects related to the equation, we refer the reader to Villani (2002) and the references therein.

From a numerical point of view, the development of grid-based methods for the Landau equation represents a challenge in scientific computing. Most of the difficulties are due to the multidimensional nature of the problem (a seven-dimensional problem, six space dimensions plus time) and to the structure of the collision integral which leads to the so-called curse of dimensionality. In addition, the numerical integration requires great care since the collision term is at the basis of the macroscopic properties of the equation.

In contrast with the Boltzmann equation, where Monte Carlo methods play a major rule in numerical simulations (see, for example, Pareschi and Russo (1999) for 
a survey on Monte Carlo methods and Cercignani et al. (1994) for some rigorous mathematical results), the construction of efficient Monte Carlo methods for longrange interactions like the Coulomb potential field is still not fully understood. Recently, important advancements in this direction have been achieved by Bobylev and Nanbu (2000), where a Monte Carlo simulation method has been derived directly from the Boltzmann equation in the grazing collision approximation. The performance of Bobylev-Nanbu scheme has been studied in detail by Wang et al. (2008). Monte Carlo methods for collisional plasmas have also been developed by Dimarco et al. (2010), while an hybrid method for accelerating the simulation of Coulomb collisions has been realized and numerically tested in Caflisch et al. (2008). In general, all these Monte Carlo method suffer from the two typical limitations of probabilistic particle methods, namely the slow convergence rate and the numerical noise in the solutions.

Therefore, it is highly desirable to develop effective grid-based methods that solve directly the partial differential (1.1). Due to the computational complexity many papers are devoted to simplified situations, like in the isotropic case (Bobylev et al. 1980). The construction of conservative and entropic schemes for the space homogeneous Landau equation has been proposed by Degond and Lucquin-Desreux (1994) and Buet and Cordier $(1998,1999)$. These schemes are built in such a way that the main physical properties are conserved at a discrete level. Positivity of the solution and discrete entropy inequality are also satisfied. However, the direct implementation of such schemes is very expensive since the computational cost increases roughly as $n^{2}$, where $n$ is the total number of parameters used to represent the distribution function in the velocity space. For this reason, several fast approximated algorithms, based on multipole expansions (Lemou 1998) or multigrid techniques (Buet and Cordier 1998), have been proposed to reduce the computational complexity. We refer also to Valentini et al. (2009), and the references therein, for different numerical approaches.

An effective way to overcome the computational complexity of the Landau equation is based on the use of spectral methods. Fourier-Galerkin methods have been recently proposed for the Boltzmann (Pareschi and Perthame 1996; Pareschi and Russo 2000a; Mouhot and Pareschi 2004; Filbet et al. 2006; Mouhot and Pareschi 2006) and the Landau (Pareschi et al. 2000a,b; Filbet and Pareschi 2002, 2003) collision operators (for related approaches in the Boltzmann case, we refer to Bobylev and Rjasanow (1997) and Gamba and Tharkabhushanam (2009)). The main advantage of spectral schemes is that they permit to obtain spectrally accurate solutions with a reduction of the quadratic cost $n^{2}$ to $n \log _{2} n$. The lack of discrete conservations in the spectral scheme (mass is preserved, whereas momentum and energy are approximated with spectral accuracy) is compensated by its higher accuracy and efficiency. A detailed comparison of the spectral scheme with the schemes proposed in Buet et al. (1997); Lemou (1998) has been done in Buet et al. (1999).

The main goal of this paper is to present efficient numerical methods for a collisional plasma by coupling a steady state preserving spectral technique (Filbet et al. 2014) for the collision term (1.3) with a Vlasov solver for the transport step (Sonnendrücker 2013). In addition, the solution of the collisional Landau term is performed with asymptotic-preserving time integration methods which, among other, permit to overcome the parabolic stiffness of the equation which relates the time step to the square of the velocity mesh ( $\mathrm{Li}$ et al. 2014). The above properties, as we will see, are essential to perform efficiently space non-homogeneous computations with high-order accuracy.

The rest of the article is organized as follows. In Sec. 2, we introduce different splitting methods to combine the Landau collisional step and the Vlasov-Poisson 
collisionless step. The fast spectral solver is then presented and modified in order to achieve the steady state preserving property. The semi-Lagrangian solvers used for the Vlasov-Poisson term are then shortly reviewed. Sec. 3 is devoted to the issue of the time discretization of the collisional phase. Efficient IMEX Runge-Kutta schemes and Exponential methods which permits to avoid the parabolic stiffness of the Landau operator are introduced. Their properties and relations with the corresponding fluid limit of the Vlasov-Landau equation are then discussed. Finally in Sec. 4, several numerical tests for space homogeneous and space non-homogeneous problems are presented. Some conclusions are then reported in Sec. 5.

\section{Numerical methods}

Here, we restrict ourselves to operator splitting-based schemes. It is well-known, in fact, that most numerical methods for collisional kinetic equation are based on a splitting in time between particle transport and collisions. A major advantage of splitting schemes is that they permit to embed a numerical method for the collision term in a pre-existing Vlasov-Poisson solver. Alternative approaches are based on the use of Implicit-Explicit Runge-Kutta schemes (Ascher et al. 1997; Pareschi and Russo 2005; Dimarco and Pareschi 2013), here we do not explore this direction and refer to Sec. 3.1.1 for a description of such methods in the collisional step.

\subsection{Splitting methods}

As it is usually done for a kinetic equation like (1.1) a simple first order time splitting is obtained considering in a small time interval $\Delta t=\left[t^{n}, t^{n+1}\right]$ the numerical solution of the space homogeneous collision phase $\mathscr{C}_{\Delta t}\left(f^{n}\right)$

$$
\left\{\begin{array}{l}
\frac{\partial f^{*}}{\partial t}=\frac{1}{\varepsilon} Q\left(f^{*}, f^{*}\right), \\
f^{*}(0, x, v)=f^{n}(x, v),
\end{array}\right.
$$

and, the transport step $\mathscr{T}_{\Delta t}\left(f^{*}\right)$

$$
\left\{\begin{array}{l}
\frac{\partial f^{* *}}{\partial t}+v \cdot \nabla_{x} f^{* *}+F(t, x) \cdot \nabla_{v} f^{* *}=0, \\
f^{* *}(0, x, v)=f^{*}(\Delta t, x, v) .
\end{array}\right.
$$

The approximated value at time $t^{n+1}$ is then given by

$$
f^{n+1}(x, v)=f^{* *}(\Delta t, x, v)=\mathscr{T}_{\Delta t}\left(\mathscr{C}_{\Delta t}\left(f^{n}\right)\right) .
$$

Higher order splitting formulas can be derived in different ways (see Hairer et al. 2010). The well-known second-order Strang splitting (Strang 1968) can be written as

$$
\mathscr{C}_{\Delta t / 2}\left(\mathscr{T}_{\Delta t}\left(\mathscr{C}_{\Delta t / 2}\left(f^{n}\right)\right)\right) \text {. }
$$

Unfortunately for splitting methods of order higher than two it can be shown that it is impossible to avoid negative time steps both in the transport as well as in the collision (Hairer et al. 2010). Higher order formulas which avoid negative time stepping can be obtained as suitable combination of splitting steps (Dia and Schatzman 1996). For example, a third-order approximation is given by

$$
\frac{2}{3}\left[\mathscr{T}_{\Delta t / 2}\left(\mathscr{C}_{\Delta t}\left(\mathscr{T}_{\Delta t / 2}\left(f^{n}\right)\right)\right)+\mathscr{C}_{\Delta t / 2}\left(\mathscr{T}_{\Delta t}\left(\mathscr{C}_{\Delta t / 2}\left(f^{n}\right)\right)\right)\right]-\frac{1}{6}\left[\mathscr{T}_{\Delta t}\left(\mathscr{C}_{\Delta t}\left(f^{n}\right)\right)+\mathscr{C}_{\Delta t}\left(\mathscr{T}_{\Delta t}\left(f^{n}\right)\right)\right],
$$


which corresponds to take a combination of symmetrized Strang and first-order splitting, whereas a fourth order scheme reads

$$
\frac{4}{3} \mathscr{C}_{\Delta t / 4}\left(\mathscr{T}_{\Delta t / 2}\left(\mathscr{C}_{\Delta t / 2}\left(\mathscr{T}_{\Delta t / 2}\left(\mathscr{C}_{\Delta t / 4}\left(f^{n}\right)\right)\right)\right)\right)-\frac{1}{3} \mathscr{C}_{\Delta t / 2}\left(\mathscr{T}_{\Delta t}\left(\mathscr{C}_{\Delta t / 2}\left(f^{n}\right)\right)\right) .
$$

Clearly, all the above splitting methods admit the symmetric formulation obtained by switching the transport and the collision operators. The crucial point is the numerical solution to (2.1) because of the presence of the collision operator and the diffusive stiffness. In the following, we first present the numerical approximation of the collision operator using a fast spectral method which is capable to preserve exactly the Maxwellian steady states. Next, we recall some basic facts concerning the discretization of the Vlasov part.

\subsection{Fast spectral methods for the Landau operator}

Spectral methods for solving the Boltzmann equation have their roots in the works of Pareschi and Perthame (1996) and Pareschi and Russo (2000a). Subsequently, their properties were studied in Pareschi and Russo (2000b) and in Filbet and Mouhot (2011). Related approaches, based on the use of the Fourier transform have been introduced by Bobylev and Rjasanow $(1997,1999)$ and Gamba and Tharkabhushanam (2009). The method has been successfully extended to the Landau equation in Pareschi et al. (2000a,b); Filbet and Pareschi $(2002$, 2003) together with the derivation of fast algorithms. On the contrary, fast algorithms for the Boltzmann case have been much more difficult to achieve (Mouhot and Pareschi 2004, 2006). Finally, the connection between the two approaches in the grazing limit has been studied in Pareschi et al. (2003); Gamba and Haack (2014).

In this section, we first recall briefly the derivation of the method for the Landau equation, the fast algorithm, and its mathematical properties (consistency and spectral accuracy). Next, following the recent result in Filbet et al. (2014), we show how to modify the spectral method in order to capture exactly the Maxwellian steady state without loosing spectral accuracy.

\subsubsection{The classical fast spectral method.}

We rewrite here the expression of the Landau integral in dimension $d \geqslant 2$ after the change of variables $q=v-v_{*}$

$$
Q(f, f)(v)=\nabla_{v} \cdot \int_{\mathbb{R}^{d}} \phi(q)\left[f(v-q) \nabla_{v} f(v)-f(v) \nabla_{q} f(v-q)\right] d q .
$$

We will assume the support of the distribution function is included in the ball $\mathscr{B}_{0}(R)$ centered in the origin and with radius $R>0$. This assumption is clearly false in general, but it is essential from a numerical point of view for any method that uses a finite velocity space for the representation of the distribution function. This is equivalent to assume that the distribution function is truncated to zero for large velocities $|v|>R$. Moreover, it is easy to prove that if $\operatorname{supp}(f(v)) \subset \mathscr{B}_{0}(R)$ then $\operatorname{supp}(Q(f, f)(v)) \subset \mathscr{B}_{0}(3 R)$.

In order to write a spectral approximation to (2.7), we can consider the distribution function $f(v)$ restricted on $[-T, T]^{d}$ with $T \geqslant 3 R$, assuming $f(v)=0$ on $[-T, T]^{d} \backslash$ $\mathscr{B}_{0}(R)$, and extend it by periodicity to a periodic function on $[-T, T]^{d}$. The lower bound for $T$ can be improved using the periodicity of the function and allowing intersections of periods, where the function $f$ is zero, to get $T \geqslant 2 R$ (Pareschi et al. $2000 \mathrm{a}, \mathrm{b})$. Therefore, aliasing errors are avoided if the integration over $\mathbb{R}^{d}$ in (2.7) is 
replaced by an integration over $\mathscr{B}_{0}(2 R)$. To simplify the notation let us take $T=\pi$ and hence $R=\pi / 2$.

We approximate the distribution by a partial sum of a Fourier series,

$$
f_{N}(t, v)=\sum_{k=-N}^{N} \hat{f}_{k}(t) e^{i k \cdot v}
$$

where $k \in \mathbb{Z}^{d}$, and we use a compact notation for the $d$-dimensional sums with $N$ the number of half modes in each direction and the $k$ th mode given by

$$
\hat{f}_{k}(t)=\frac{1}{(2 \pi)^{d}} \int_{[-\pi, \pi]^{d}} f(t, v) e^{-i k \cdot v} d v .
$$

Now, substituting the approximation $f_{N}(t, v)$ in the operator $Q^{R}$, where $Q^{R}$ is the Landau operator with cut-off over the relative velocity in the ball $\mathscr{B}_{0}(\pi)$ we obtain

$$
Q^{R}\left(f_{N}, f_{N}\right)=\sum_{k=-2 N}^{2 N} \hat{Q}_{k}^{R} e^{i k \cdot v}
$$

with

$$
\hat{Q}_{k}^{R}=\sum_{\substack{l+m=k \\ l, m=-N}}^{N} \hat{f}_{l} \hat{f}_{m} \hat{\beta}(l, m),
$$

where $\hat{\beta}(l, m)=\hat{B}(l, m)-\hat{B}(m, m)$, and the Landau kernel modes $\hat{B}(l, m)$ are given by

$$
\hat{B}(l, m)=\int_{\mathscr{B}_{0}(\pi)}|g|^{\gamma+2}\left[l^{2}-\left(l \cdot \frac{g}{|g|}\right)^{2}\right] e^{i g \cdot m} d g .
$$

It can be proved that the coefficients $\hat{B}(l, m)$ are scalar quantities independent on the function $f$ which depend on $|l+m|$ and $|l-m|$ only (Pareschi et al. 2000a,b). Moreover, for inverse power laws, taking $C=(4 \pi)(\pi)^{d+2+\gamma}$ we have the bound

$$
|\hat{B}(l, m)| \leqslant C \frac{d N^{2}}{d+2+\gamma} .
$$

Note that $\hat{B}(l, m)$ grow with $N^{2}$, and this is the cause of the stiffness observed in the time integration of the equation (Filbet and Pareschi 2002, 2003). This reflects the fact that the Landau equation suffers of the stiffness typical of diffusion equations. Stability condition of grid-based methods requires that the time step scales with the square of the velocity mesh size. Time integration methods that overcome this problem will be the subject of Sec. 3 .

Let now $\mathscr{P}_{N}: L^{2}\left([-\pi, \pi]^{d}\right) \rightarrow I P^{N}$ be the orthogonal projection upon the space of trigonometric polynomials of degree $N$ in $v, I^{N}$ in the inner product of $L^{2}\left([-\pi, \pi]^{d}\right)$. Then, the spectral method for the collision step (2.1), where we take $\varepsilon=1$ for simplicity, can be written in equivalent form as

$$
\frac{\partial f_{N}}{\partial t}=Q_{N}^{R}\left(f_{N}, f_{N}\right)
$$

with initial data $f_{N}(v, t=0)=f_{0, N}(v)$ and

$$
Q_{N}^{R}\left(f_{N}, f_{N}\right):=\mathscr{P}_{N} Q^{R}\left(f_{N}, f_{N}\right),
$$

where $Q^{R}\left(f_{N}, f_{N}\right)$ is given by (2.9). 
It is easy to verify that the spectral method preserves mass whereas variations of momentum and energy are controlled by the spectral accuracy (Pareschi and Russo 2000a; Pareschi et al. 2000b). By the arguments in Pareschi et al. (2000a) it is possible to prove consistency and spectral accuracy of the method

THEOREM 2.1. The spectral approximation of the collision operator defined by (2.9)(2.13) is such that the following properties hold

(i) (consistency) Let $f \in H_{p}^{2}\left([-\pi, \pi]^{d}\right)$, then $\forall r \geqslant 0$

$$
\left\|Q^{R}(f, f)-Q_{N}^{R}\left(f_{N}, f_{N}\right)\right\|_{2} \leqslant C\left(\left\|f-f_{N}\right\|_{H_{p}^{2}}+\frac{\left\|Q^{R}\left(f_{N}, f_{N}\right)\right\|_{H_{p}^{r}}}{N^{r}}\right),
$$

where $C$ depends on $\|f\|_{2}$.

(ii) (spectral accuracy) Let $f \in H_{p}^{r}\left([-\pi, \pi]^{d}\right), r \geqslant 2$ then

$$
\left\|Q^{R}(f, f)-Q_{N}^{R}\left(f_{N}, f_{N}\right)\right\|_{2} \leqslant \frac{C}{N^{r-2}}\left(\|f\|_{H_{p}^{r}}+\left\|Q^{R}\left(f_{N}, f_{N}\right)\right\|_{H_{p}^{r}}\right) .
$$

In the previous theorem, $H_{p}^{r}$ denotes the Sobolev space of periodic functions up to the $r$ th order derivative over $[-\pi, \pi]^{d}$. No information are available on the discrete equilibrium states, the decay of the numerical entropy and the preservation of positivity. We mention that spectral schemes where conservation are enforced by some kind of renormalization procedure have been developed in (Pareschi and Perthame 1996; Bobylev and Rjasanow 1997; Gamba and Tharkabhushanam 2009).

\subsubsection{A fast summation method.}

First, let us note that a direct computation of (2.10) has the same $O\left(n^{2}\right), n=N^{d}$, cost of a conventional finite difference discretization applied to the Landau equation.

On the other hand we can rewrite (2.10) as

$$
\sum_{m=-N}^{N} \hat{f}_{k-m} \hat{f}_{m} \hat{B}(k-m, m)-\sum_{m=-N}^{N} \hat{f}_{k-m} \hat{f}_{m} \hat{B}(m, m), \quad k=-N, \ldots, N .
$$

Clearly, the second sum is a convolution sum and thus transform methods allow this term to be evaluated in $O\left(n \log _{2} n\right)$ operations. For the details of the implementation of this standard technique for spectral methods, we refer the reader to Canuto et al. (1988). Hence, the most expensive part of the computation is represented by the first sum which in general cannot be evaluated with fast algorithms.

In the case of the Landau equation, however, $\hat{B}(l, m)$ splits as

$$
\hat{B}(l, m):=l^{2} \tilde{F}(m)-\sum_{p, q=1}^{d} l_{p} l_{q} I_{p q}(m)=l^{2} \tilde{F}(m)-l \mathscr{I}(m) l^{T},
$$

where $l^{T}$ denotes the transpose of the vector $l, \mathscr{I}=\left(I_{p q}\right)$ is a $d \times d$ symmetric matrix

$$
\begin{aligned}
\tilde{F}(m) & =\int_{\mathscr{B}_{0}(\pi)}|g|^{2+\gamma} e^{i g \cdot m} d g, \\
I_{p q}(m) & =\int_{\mathscr{B}_{0}(\pi)}|g|^{\gamma} g_{p} g_{q} e^{i g \cdot m} d g, \quad p, q=1, \ldots, d .
\end{aligned}
$$

Thus, we can write

$$
\hat{\psi}(l, m)=l^{2} \tilde{F}(m)-l \mathscr{I}(m) l^{T}-\hat{B}_{L}(m, m) .
$$


The resulting scheme requires the evaluation of $2 d+2$ convolution sums (the number of distinct elements of $\mathscr{I}$ plus two single convolution sums for $\tilde{F}(m)$ and $\hat{B}_{L}(m, m)$ ). Hence, the overall cost of the scheme is only $O\left(n \log _{2} n\right)$.

For the implementation of the algorithm, we need to evaluate the quantities (2.14)(2.15). For simplicity, we will treat here only the two-dimensional case $v \in \mathbb{R}^{2}$. We have

$$
\begin{aligned}
& I_{11}(m)=\frac{1}{2}\left[F(|m|)+\frac{m_{1}^{2}-m_{2}^{2}}{|m|^{2}} G(|m|)\right], \\
& I_{22}(m)=\frac{1}{2}\left[F(|m|)-\frac{m_{1}^{2}-m_{2}^{2}}{|m|^{2}} G(|m|)\right], \\
& I_{12}(m)=I_{21}(m)=\frac{m_{1} m_{2}}{|m|^{2}} G(|m|),
\end{aligned}
$$

where

$$
\tilde{F}(m)=F(|m|)=2 \pi \int_{0}^{\pi} r^{\gamma+3} J_{0}(|m| r) d r,
$$

with $J_{0}$ the Bessel function of order 0 and

$$
G(|m|)=\int_{0}^{\pi} r^{\gamma+3} \int_{0}^{2 \pi} \cos (|m| r \cos \phi) \cos (2 \phi) d \phi d r
$$

Thus, the computation reduces simply to the computation of two one-dimensional integrals $F(|m|)$ and $G(|m|)$. These quantities can be computed very accurately once and then stored in two bidimensional arrays. A similar reduction can be performed in the full three-dimensional case.

\subsubsection{The steady state preserving spectral method.}

A major drawback of the method just described is the lack of conservations and, in particular, the incapacity of the scheme to preserve the Maxwellian steady states of the system. Renormalization strategies to recover the conservations have been proposed by various authors (Pareschi and Perthame 1996; Bobylev and Rjasanow 1997; Gamba and Tharkabhushanam 2009), however these do not guarantee the Maxwellian behaviour of the solution. A modified spectral method which is capable to overcome this difficulty has been proposed recently in Filbet et al. (2014). Here, we describe the method in the case of the Landau operator.

Let us start from the decomposition

$$
f=M+g,
$$

with $M$ the local Maxwellian equilibrium and $g$ such that $\int_{\mathbb{R}^{3}} g \phi d v=0, \phi=1, v,|v|^{2}$. When inserted into the Landau operator gives

$$
Q(f, f)=\mathscr{L}(M, g)+Q(g, g),
$$

where $\mathscr{L}(M, g)=Q(g, M)+Q(M, g)$ and we used the fact that

$$
Q(M, M)=0 .
$$

Note that the steady state of $(2.21)$ is given by $g \equiv 0$. To illustrate the method let us consider now the space homogeneous equation that we rewrite using the micro-macro 
decomposition as

$$
\begin{aligned}
\frac{\partial g}{\partial t} & =\mathscr{L}(M, g)+Q(g, g), \\
f & =M+g .
\end{aligned}
$$

Using the same notations of the previous section we can write the Fourier-Galerkin approximation

$$
\begin{aligned}
\frac{\partial g_{N}}{\partial t} & =\mathscr{L}_{N}^{R}\left(M_{N}, g_{N}\right)+Q_{N}^{R}\left(g_{N}, g_{N}\right), \\
f_{N} & =M_{N}+g_{N},
\end{aligned}
$$

where

$$
M_{N}:=\mathscr{P}_{N} M, \quad g_{N}:=\mathscr{P}_{N} g, \quad \mathscr{L}_{N}^{R}\left(M_{N}, g_{N}\right):=\mathscr{P}_{N} \mathscr{L}^{R}\left(M_{N}, g_{N}\right)
$$

It is immediate to see that $g_{N} \equiv 0$ is an admissible local equilibrium of the spectral scheme (2.24) and therefore $f_{N}=M_{N}$ is a local equilibrium state.

It is interesting to observe that the only difference between scheme (2.24) and the usual spectral method developed on the original formulation

$$
\begin{aligned}
\frac{\partial f_{N}}{\partial t} & =\frac{\partial g_{N}}{\partial t} \\
& =Q_{N}^{R}\left(f_{N}, f_{N}\right), \\
& =\mathscr{L}_{N}^{R}\left(M_{N}, g_{N}\right)+Q_{N}^{R}\left(g_{N}, g_{N}\right)+Q_{N}^{R}\left(M_{N}, M_{N}\right)
\end{aligned}
$$

is due to the constant (in time) term

$$
Q_{N}^{R}\left(M_{N}, M_{N}\right) \neq 0,
$$

which is spectrally close to 0 since from Theorem 2.1 for $f=M$ we obtain

LEMMA 2.1. For $r \geqslant 2$ we have

$$
\left\|Q_{N}^{R}\left(M_{N}, M_{N}\right)\right\|_{2} \leqslant \frac{C}{N^{r-2}}\left(\|M\|_{H_{p}^{r}}+\left\|Q^{R}\left(M_{N}, M_{N}\right)\right\|_{H_{p}^{r}}\right), \quad \forall r \geqslant 2 .
$$

From the above estimate spectral accuracy of the steady state preserving method follows immediately. Finally, from the computational viewpoint the new method can be implemented through the same fast algorithm just described, simply by removing the constant term (2.27) in the usual spectral method. In this way, we avoid the accumulation of errors and can take advantage of the fact that $f_{N}=M_{N}$ is the steady state of the numerical scheme.

\subsection{Semi-Lagrangian schemes for the Vlasov equation}

In this paragraph, we give a short overview of semi-Lagrangian method, we employ to discretize the left-hand side of the Vlasov-Landau equation (1.1). The semiLagrangian methods use, as classical finite volume methods, a fixed mesh in space and in velocity space but they take advantage of the knowledge of the exact solution of the linear transport process which characterizes the kinetic equations. In general, the advantages of these methods are related to the fact that they can be quite easily designed in order to possess many desired properties such as positivity, physical conservations and robustness when dealing with large velocities. More in details, they do not suffer of the time step restrictions which are typical of other classes of schemes, thus the CFL conditions are normally only imposed in order to keep errors sufficiently small. 
Several different semi-Lagrangian approaches have been developed in the past Boris and Book (1973); Cheng and Knorr (1976) and more recently Carrillo and Vecil (2007); Qiu and Shu (2011); Ayuso et al. (2011); Heath et al. (2012); Cheng et al. (2012); Crouseilles et al. (2010); Sonnendrücker et al. (1999); Filbet et al. (2001); Valentini et al. (2007) which address the particular problem of solving Vlasov type kinetic equations by this kind of techniques. Here, we recall the basic features of the method used and we refer to the above references and the recent introductory notes by Sonnendrücker (2013) for a more complete overview of this class of methods.

We consider the left-hand side of (1.1)

$$
\frac{\partial f}{\partial t}+v \cdot \nabla_{x} f+E(t, x) \cdot \nabla_{v} f=0, \quad v \in \mathbb{R}^{3}, x \in \Omega \subset \mathbb{R}^{3} .
$$

The above equation can be solved by means of semi-Lagrangian methods by employing again time splitting techniques which separates the transport in the physical space by the transport in the velocity space. A first-order splitting scheme reads as

$$
\left\{\begin{array}{l}
\frac{\partial f^{*}}{\partial t}+v \cdot \nabla_{x} f^{*}=0, \\
f^{*}(0, x, v)=f^{n}(x, v),
\end{array}\right.
$$

and

$$
\left\{\begin{array}{l}
\frac{\partial f^{* *}}{\partial t}+E^{* *} \cdot \nabla_{v} f^{* *}=0, \\
f^{* *}(0, x, v)=f^{*}(\Delta t, x, v),
\end{array}\right.
$$

with $f^{n+1}(x, v)=f^{* *}(x, v)$ and $E^{n+1}(x)=E^{* *}(x)=-\nabla_{x} \phi^{* *}(x)$. In the above scheme, the electric potential is computed by solving the Poisson equation after the transport step, which means $-\Delta_{x} \phi^{* *}(x)=\int_{\mathbb{R}^{3}} f^{* *}(0, x, v) d v=\int_{\mathbb{R}^{3}} f^{*}(\Delta t, x, v) d v$. To solve the Poisson equation, we used a classical finite difference fourth-order discretization. As in the case of collisions, high-order splitting can be used as well to increase the accuracy with respect to time. In practice, in the numerical test section we employed a second-order Strang splitting (2.4) and a fourth-order time splitting (2.6) method. The most common reconstruction techniques found in the literature are cubic splines, Hermite or Lagrange polynomials. In our computations, we employed cubic spline reconstructions. In the one-dimensional setting the cubic spline interpolation $f_{\Delta x}$ of a function $f$ is defined by a cubic function $f_{\Delta x} \in \mathbb{P}_{3}(I)$ on each interval $I$ of length $\Delta x$ in which the space is discretized with $f_{\Delta x}\left(x_{j}\right)=f\left(x_{j}\right)$ and $f_{\Delta x} \in C^{2}(I)$. Writing $f_{\Delta x}$ using the cubic B-spline basis we obtain

$$
f_{\Delta x}(x)=\sum_{h=0}^{N-1} a_{h} S^{3}\left(x-x_{h}\right),
$$

where $N$ are the number of points of the mesh and the coefficients $a_{h}$ are given by the interpolation conditions

$$
f\left(x_{j}\right)=f_{\Delta x}\left(x_{j}\right)=\sum_{h=0}^{M-1} a_{h} S^{3}\left(x_{j}-x_{h}\right),
$$


while the cubic B-spline basis is defined by

$$
S^{3}(x)=\frac{1}{6} \begin{cases}\left(2-|x| / \Delta x^{3}\right), & \text { if } \Delta x<|x|<2 \Delta x \\ 4-6(|x| / \Delta x)^{2}+3(|x| / \Delta x)^{3}, & \text { if } 0<|x|<\Delta x \\ 0, & \text { otherwise. }\end{cases}
$$

\section{A penalization technique for efficient time integration}

In this section, we focus on the development of efficient numerical time integrators for solving the space homogeneous Landau equation, the full solution of the collisional Vlasov equation being obtained by means of splitting techniques as the ones described in Sec. 2.1. The time discretization of the collision term brings new numerical difficulties to the problem. They can be summarized by

(1) Parabolic stiffness: the Landau operator is generically a diffusive operator, and if one uses explicit methods, typically a time constraint $\Delta t \sim \Delta v^{2}$ is expected.

(2) Strong collisions regimes: When one deals with strong collisional regimes the Knudsen number $\varepsilon$ tends to 0 which leads to a fast convergence to the equilibrium state. In these situations, the stability of standard explicit methods is related to time steps of order $\Delta t \sim \varepsilon$.

These two issues combined cause typically time steps restrictions of type $\Delta t \sim \varepsilon \Delta v^{2}$, which determine a tremendous computational cost in practical computations. In the following, we present two efficient strategies which permit to overcome these difficulties leading to high order in time schemes. As the time scale imposed by $\varepsilon$ always comes with the time scale imposed by $\Delta v^{2}$, numerically one could simply set $\varepsilon=1$ and derive the schemes in this general setting.

\subsection{Removing the parabolic stiffness}

The problem we address is the following

$$
\partial_{t} f=Q(f), \quad v \in \mathbb{R}^{3} .
$$

with $Q$

$$
Q(f)=\nabla_{v} \cdot \int_{\mathbb{R}^{3}} \Phi\left(v-v^{*}\right)\left[f\left(v^{*}\right) \nabla_{v} f(v)-f(v) \nabla_{v^{*}} f\left(v^{*}\right)\right] d v^{*} .
$$

The Landau operator is a diffusive-type operator with eigenvalues expanding to infinity analytically and to $\mathcal{O}\left(1 / \Delta v^{2}\right)$ numerically. This causes the so-called parabolic stiffness $\Delta t \sim(\Delta v)^{2}$ which one encounters if standard explicit schemes are employed. On the other hand, standard implicit methods which exhibit larger stability regions and thus allow bigger time steps, cannot be used in this situation, since the numerical complexity related to the discretization of $Q$ makes impossible, in practice, its inversion.

In order to construct schemes which permit to avoid the stiffness of the collision operator and its numerical inversion, we introduce a suitable penalization operator which is easier to invert with respect to the original operator. In order to guarantee an efficient, high order and consistent with the limit $\varepsilon \rightarrow 0$ scheme, the penalization operator $P$ needs to satisfy the following criteria:

- $P$ should share the same equilibrium state of the original operator $Q$, i.e. the functions $f$ such that $Q(f)=0$ are also such that $P(f)=0$. This property guarantees the correct and stable solution for infinite times. 
- $P$ should preserve the first three moments, that is,

$$
\int\left[1, v, v^{2}\right]^{T} P(f) \mathrm{d} v=0 .
$$

This requirement is imposed in order to preserve mass, momentum and energy in the evolution of the system as the original operator $Q$ does.

- $P$ should have a diffusive character which permits to capture the diffusive behaviors of the original operator $Q$.

- $P$ should be easy to invert.

The simplest operator which fulfills all the above characteristics is the so-called Fokker-Planck operator:

$$
P(f)=\mu \nabla_{v} \cdot\left(M \nabla_{v}\left(\frac{f}{M}\right)\right) .
$$

Here, $\mu$ is a numerical constant, and $M$ is the Maxwellian function that shares the first three moments with $f$. This operator has been recently used as a penalization operator in Jin and Yan (2011) for constructing asymptotically stable schemes.

Thus, the original (3.1) is modified in

$$
\partial_{t} f=\underbrace{[Q(f)-P(f)]}_{\text {non-stiff }}+\overbrace{P(f)}^{\text {stiff }} \doteq R(f)+P(f) .
$$

Now, thanks to this reformulation, the first term, denoted by $R$, has most of its parabolic scale canceled out, and is expected to be non-stiff, or comparably less stiff, while the second term, albeit diffusive, is easy to be inverted. Starting from the above reformulation in the following we propose two different approaches both leading to accurate and stable time discretizations which avoid the parabolic stiffness: Implicit-Explicit Runge-Kutta (IMEX) methods, and exponential RungeKutta (ERK) methods. We conclude this part with some comments about the choice of the parameter $\mu$.

ReMARK 3.1 (ON THE CHOICE OF $\mu$ ). As we will made more clear after the introduction of the details of the time integrators, larger is $\mu$ more stable is the scheme. On the other hand, large values of $\mu$ causes largers errors. This is due to the fact that larger is $\mu$ more important becomes the contribution of the penalization operator $P$ with respect to the original operator $Q$. Thus, as suggested in Jin and Yan (2011) a good balance between stability and numerical error can be

$$
\mu=\mu_{0} \max _{v} \Lambda\left(D_{\Phi}(f)\right) .
$$

Here, $\mu_{0}$ is a constant such that $\mu_{0}>\frac{1}{2}$ while $\Lambda\left(D_{\Phi}\right)$ is the spectral radius of the positive symmetric matrix $D_{\Phi}$, with $D_{\Phi}(f)$ defined by

$$
D_{\Phi}(f)=\int \Phi\left(v-v_{*}\right) f_{*} d v_{*} .
$$

We remind to Jin and Yan (2011) for details about this particular choice and other possible choices for $\mu$.

\subsubsection{IMEX-RK scheme.}

The idea behind the IMEX-RK schemes is to construct a framework which permit to discretize with high order of accuracy a given equation treating with different 
Runge-Kutta schemes the different terms which appears in the equation. Thus, in the case of (3.5) the idea is to treat explicitly the non-stiff part (the part due to the difference of the original operator and the penalization one, $R$ in (3.1)), while to treat implicitly the easier to invert penalized operator. The IMEX-RK strategy dates back to Ascher et al. (1997), where they have been employed for parabolic partial differential equations, successively they were extended in Pareschi and Russo (2005) to hyperbolic relaxation systems. Early examples also include Jin (1995) and Caflisch et al. (1997). Recently they have been designed to achieve asymptotic preservation for the Boltzmann equation in the fluid limit without requiring the inversion of the collision operator, see Filbet and Jin (2010) and Dimarco and Pareschi (2012, 2013).

One of the simplest example of IMEX-RK is obtained connecting the forward and backward Euler method leading to a first order accurate and uniformly stable scheme

$$
\frac{f^{n+1}-f^{n}}{\Delta t}=R^{n}+P^{n+1} .
$$

As in the homogeneous case $M$ is constant we can rewrite the above equation as

$$
(\mathbb{I}-\Delta t \mathbb{P}) f^{n+1}=f^{n}+\Delta t R^{n},
$$

with $R^{n} \doteq R\left(f^{n}\right)=Q\left(f^{n}\right)-P\left(f^{n}\right)$ and $P^{n+1}=P\left(f^{n+1}\right)=\mathbb{P} \cdot f^{n+1}$.

Higher order of accuracy can be obtained by properly combining two Runge-Kutta schemes one for the explicit and one for the implicit part. The schemes are, in general, defined thanks to the so-called double Butcher tableaux

$$
\frac{\tilde{c} \mid \tilde{A}}{\mid \tilde{w}^{T}} \quad \frac{c \mid A}{\mid w^{T}},
$$

in which $\tilde{A}$ and $A$ represent the coefficients of respectively the explicit and the implcit Runge-Kutta scheme while $\tilde{w}$ and $w$ are the so called coefficients of the numerical solution. Thus, an IMEX-RK scheme reads

$$
\left\{\begin{array}{l}
f^{(i)}=f^{n}+\Delta t \sum_{j=1}^{i-1} \tilde{a}_{i j} R^{(j)}+\Delta t \sum_{j=1}^{v} a_{i j} P^{(j)} \\
f^{n+1}=f^{n}+\Delta t \sum_{i=1}^{v} \tilde{w}_{i} R^{(i)}+\Delta t \sum_{i=1}^{v} w_{i} P^{(i)},
\end{array} .\right.
$$

where $f^{(i)}$ are commonly called the stages of the R-K scheme. Since the first R-K scheme is explicit, $\tilde{A}$ is lower-triangular. On the other hand, $A$ is in general a full matrix. This indicates the implicit character of the second R-K scheme. However, in most of the applications, one restricts to diagonally implicit Runge-Kutta (DIRK) schemes, that is $a_{i j}=0$, for $j>i$. This is sufficient to show that $R$ is really treated explicitly in the global IMEX Runge-Kutta scheme. In a compact form, this class of scheme can be rewritten as

$$
\left\{\begin{array}{l}
F=f^{n} e+\Delta t \tilde{A} \cdot R(F)+\Delta t A \cdot P(F) \\
f^{n+1}=f^{n}+\Delta t \tilde{w}^{T} \cdot R(F)+\Delta t w^{T} \cdot P(F)
\end{array},\right.
$$

where the notations used above are

$$
\begin{aligned}
& e=(1, \ldots, 1)^{T}, \quad F=\left(f^{(1)}, \ldots, f^{(v)}\right)^{T}, \quad R(F)=\left[R\left(f^{(1)}\right), \ldots, R\left(f^{(v)}\right)\right]^{T}, \\
& P(F)=\left[P\left(f^{(1)}\right), \ldots, P\left(f^{(v)}\right)\right]^{T}=\left[\mathbb{P} \cdot f^{(1)}, \mathbb{P} \cdot f^{(2)}, \ldots \mathbb{P} \cdot f^{(v)}\right] .
\end{aligned}
$$

Again, we point out that $P\left(f^{(i)}\right)=\mathbb{P} \cdot f^{(i)}$ due to the fact that $M$ is constant in the homogeneous case and the same holds for the full Vlasov equation thanks to the 
splitting approach. Finally, as expected, we point out that the two Butcher tableaux are not decoupled, in fact in order to get a prescribed order of accuracy coupled order conditions should be satisfied in addition to the traditional order conditions given by the classical Runge-Kutta method. We refer the readers to Ascher et al. (1997); Pareschi and Russo (2005) and more recently to Dimarco and Pareschi (2013) for details.

For a detailed analysis of the stability properties of the penalized IMEX-RK schemes in the context of the Landau operator we remind to Li et al. (2014).

\subsubsection{Exponential Runge-Kutta method.}

The exponential Runge-Kutta (ERK) methods represent an alternative approach with respect to the IMEX-RK methods which permit to get uniformly stable schemes which avoid the parabolic stiffness of the Landau operator. A general picture of ERK methods applied to parabolic PDE can be found in Hochbruck and Ostermann (2010), in the context of kinetic equations, these schemes were initially used by Dimarco and Pareschi (2011) for constructing asymptotic preserving schemes for the homogeneous Boltzmann equation, and successively extended to the inhomogeneous case by $\mathrm{Li}$ and Pareschi (2014), while ERK methods applied to multispecies kinetic equations and to the quantum case can be found in Li and Yang (2014) and Hu et al. (2014).

As for the IMEX-RK case, the idea consists in reformulating the homogeneous Landau (3.1) in such a way that the use of classical explicit Runge-Kutta method onto the modified equation makes the schemes uniformly stable for all possible choices of $\Delta v$ and $\Delta t$. The reformulation employed is the following:

$$
\partial_{t}\left(e^{-t \mathbb{P}} f\right)=-\mathbb{P}\left[e^{-t \mathbb{P}} f\right]+e^{-t \mathbb{P}}[Q(f)]=e^{-t \mathbb{P}}[Q(f)-\mathbb{P} \cdot f],
$$

where we used the following property of the Fokker-Planck operator $P(f)=\mathbb{P} \cdot f$

$$
e^{t \mathbb{P}} f=\left(\mathbb{I}+t \mathbb{P}+\frac{t^{2}}{2} \mathbb{P}^{2}+\cdots\right) f
$$

with $e^{t \mathbb{P}}$ a linear operator on $f$ which commutes with $\mathbb{P}$ since in the homogeneous case the Maxwellian distribution is a constant function. Let observe that the same properties can be used in the non-homogeneous case thanks to a splitting approach between the Vlasov terms and the Landau ones which causes the Maxwellian distribution to be considered constant during the solution of the collision step.

Now, using for instance the forward Euler scheme with the reformulated (3.13) leads to

$$
e^{-\Delta t \mathbb{P}} f^{n+1}=f^{n}+\Delta t\left(Q^{n}-P^{n}\right) .
$$

This can be rewritten, since $M$ is constant, as

$$
f^{n+1}-M=e^{\Delta t \mathbb{P}}\left(f^{n}-M\right)+\Delta t e^{\Delta t \mathbb{P}}\left(Q^{n}-P^{n}\right) .
$$

From the above equation one can observe that since all the eigenvalues of $\mathbb{P}$ lie on the negative side of the real line, and the one with biggest absolute value is about $\frac{1}{\Delta v^{2}}$, as $\Delta v \rightarrow 0$, the numerical solution is bounded for any choices of $\Delta t$. Moreover, the exponential term pushes the two terms on the right hand side of (3.16) to zero, leading to the convergence of $f$ to the Maxwellian state $M$. 
Higher order schemes (of $v$ th order) can be derived applying directly a standard $v$ th order Runge-Kutta method to the reformulated (3.13). They read

$$
\left\{\begin{array}{l}
e^{-c_{i} h \mathbb{P}} f^{(i)}=f^{n}+\Delta t \sum_{j=1}^{i-1} a_{i j} e^{-c_{j} \Delta t \mathbb{P}}\left(Q^{(j)}-P^{(j)}\right), \\
e^{-h \mathbb{P}} f^{n+1}=f^{n}+\Delta t \sum_{i=1}^{v} w_{i} e^{-c_{i} \Delta t \mathbb{P}}\left(Q^{(i)}-P^{(i)}\right),
\end{array}\right.
$$

where $f^{(i)}$ stands for the estimation of $f$ at time substage $t=t^{n}+c_{i} \Delta t$ with $a_{i j}$, $w_{i}$, and $c_{i}$ the standard Runge-Kutta coefficients. Since the $\mathrm{R}-\mathrm{K}$ scheme is explicit at each stage of the scheme, the operator $Q$ is evaluated using the data from the previous stages, i.e. explicitly. On the other hand, the exponential operator $e^{\Delta t \mathbb{P}}$ should be evaluated at each stage of the Runge-Kutta procedure. This computation, which represents the most costly parts of the entire procedure, can be done through different methods, some strategies are suggested for instance in Moler and Loan (1978). Among the different possibilities, we choose to compute the exponential operator through an expansion up to the $v$ th order as written in (3.14). In fact, since the entire time integrator is $v$ th order, the exponential matrix needs to be computed with the same order of accuracy if a theoretical $v$ th order scheme wants to be derived.

More in details following Jin and Yan (2011), we start by introducing a new operator $\tilde{P}$

$$
\tilde{P} h=\frac{1}{\sqrt{M}} \nabla_{v} \cdot\left(M \nabla_{v}\left(\frac{h}{\sqrt{M}}\right)\right),
$$

which is related to the original operator by

$$
P(f)=\sqrt{M} \tilde{P}\left(\frac{f}{\sqrt{M}}\right) .
$$

This new operator shares the same symmetric properties of the original operator $P$ and consequently it turns into a symmetric and positive definite matrix once discretized. These properties are very important from the computational point of view since these matrices are much less costly to invert. Then, similarly to the original operator, we have an analogous exponential expansion for $\widetilde{P}$

$$
e^{t P} f=\sqrt{M} e^{t \tilde{P}} \frac{f}{\sqrt{M}} .
$$

Thus, in practice, we discretize $\tilde{P}$, and then we transform back to $P$ by using (3.19). This can be directly checked to be enough for symmetry to be preserved. For instance, the discretization of $\tilde{P}$ in one dimension reads

$$
\begin{aligned}
(\tilde{\mathbb{P}} h)_{j}= & \frac{1}{(\Delta v)^{2}} \frac{1}{\sqrt{M_{j}}}\left\{\sqrt{M_{j} M_{j+1}}\left(\left(\frac{h}{\sqrt{M}}\right)_{j+1}-\left(\frac{h}{\sqrt{M}}\right)_{j}\right)\right. \\
& \left.-\sqrt{M_{j} M_{j-1}}\left(\left(\frac{h}{\sqrt{M}}\right)_{j}-\left(\frac{h}{\sqrt{M}}\right)_{j-1}\right)\right\} \\
= & \frac{1}{(\Delta v)^{2}}\left(h_{j+1}-\frac{\sqrt{M_{j+1}}+\sqrt{M_{j-1}}}{\sqrt{M_{j}}} h_{j}+h_{j-1}\right) .
\end{aligned}
$$

which can be straightforwardly extended to the multidimensional case. 
As for the IMEX-RK case, for a detailed analysis of the stability properties of the ERK schemes in the context of the Landau operator we remind to Li et al. (2014).

Remark 3.2 (ON THE FLUID LIMIT). When the collision term is very strong, one can show that at least formally the Vlasov-Landau equation (1.1) converges to fluid limit, namely the equation

$$
\frac{\partial f}{\partial t}+v \cdot \nabla_{x} f+E(t, x) \cdot \nabla_{v} f=\frac{1}{\varepsilon} Q(f, f), \quad v \in \mathbb{R}^{3}, x \in \Omega \subset \mathbb{R}^{3}
$$

is asymptotically equivalent to the Euler-Poisson equation

$$
\left\{\begin{array}{l}
\partial_{t} \rho+\nabla_{x} \cdot(\rho u)=0, \\
\partial_{t}(\rho u)+\nabla_{x} \cdot(\rho u \otimes u)+\nabla_{x} p=\rho E, \\
\partial_{t}(\rho e)+\nabla_{x} \cdot(\rho e+p) u=\rho u E
\end{array}\right.
$$

with

$$
[\rho, \rho u, \rho e]^{T}=\int\left[1, v, v^{2} / 2\right]^{T} f(v) \mathrm{d} v,
$$

and $p$ the gas pressure with $p=\rho R T$.

Thus, another interesting topic is about numerical methods which are able capture the above fluid limit when the Knudsen number is pushed to zero while the discretization parameters, i.e. time step and meshes, are kept fixed. The development of numerical methods designed to satisfy this property, called asymptotic preserving, has been the object of several papers, see for instance Dimarco and Pareschi (2011); Jin (1999); Filbet and Jin (2010) and the recent review papers by Jin (2012); Degond (2014); Pareschi and Russo (2011) and Dimarco and Pareschi (2014). The important feature shared by these techniques is that the resulting schemes are unconditionally stable and capture the asymptotic limit automatically without resolving the small time scales.

Thus, one can show that the Penalized Exponential Runge-Kutta and IMEX-RK methods presented may benefit of this additional property under several hypothesis on the type of RK scheme employed. In other words, this class of scheme is able to capture with high-order accuracy the fluid limit without any time or mesh step limitation when the Knudsen number goes to zero. We remind to Li et al. (2014) for details.

\section{Numerical results}

\subsection{Space homogeneous problems}

In this subsection, we perform several numerical tests in the spatially homogeneous case. The collision term $Q(f)$ is computed using the steady state preserving spectral method described in Sec. 2 where we left out the transport terms. In all the numerical tests, the computational domain is two dimensional in the velocity space: [ $\left.-v_{\max }, v_{\max }\right]^{2}$ with $v_{\max }=6$. We use $N_{v}=64$ grid points in each velocity direction. For the numerical integrator, we use the ERK or the IMEX-RK methods presented 
in Sec. 3.1. In the first case the standard Butcher tableaux below have been employed

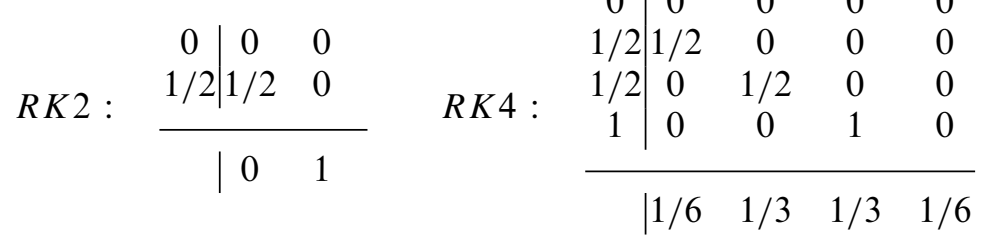

while for the IMEX-RK methods, one needs to prescribe two Butcher tableaux, one for the explicit and one for the implicit part. The schemes are

(a) First-order IMEX (Forward Euler and Backward Euler)

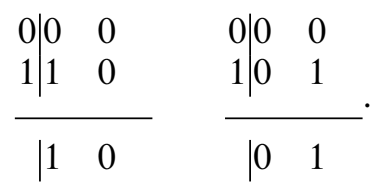

\begin{tabular}{|c|c|c|c|c|}
\hline \begin{tabular}{l|l}
0 & 0
\end{tabular} & 0 & 0 & 0 & 0 \\
\hline $1 / 2 \mid 1 / 2$ & 0 & 0 & $1 / 2$ & $1 / 2$ \\
\hline \begin{tabular}{l|l}
1 & $1 / 2$
\end{tabular} & $1 / 2$ & 0 & 1 & 0 \\
\hline & & & & 0 \\
\hline
\end{tabular}

(b) Second-order IMEX (Midpoint Rule and Trapezoidal Rule)

(c) Third-order IMEX BPR-(3,5,3)

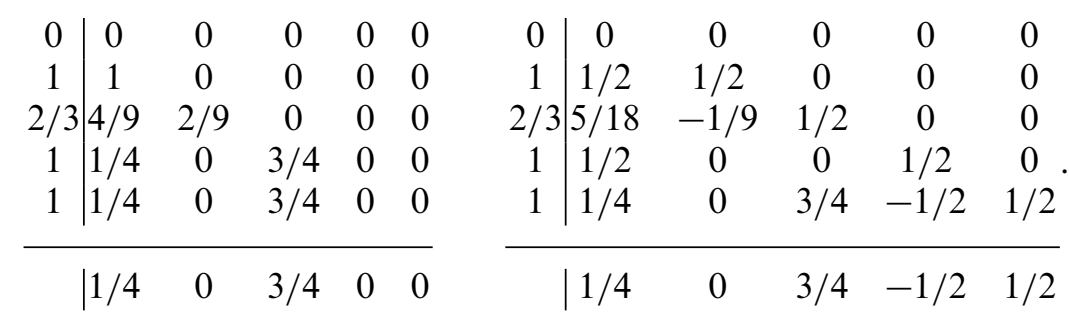

\subsubsection{Test 1: equilibrium test.}

In this first example, our aim is to check if the numerical integrator is able to capture the equilibrium state and to remain in this state for infinite time. This example is the analogous of test 2 in Filbet and Pareschi (2003). The initial distribution is a summation of two Gaussian distributions

$$
f(v, t=0)=\frac{\rho}{4 \pi T}\left(\exp \left(-\frac{(v-u)^{2}}{2 T}\right)+\exp \left(-\frac{(v+u)^{2}}{2 T}\right)\right),
$$

where the macroscopic quantities are given by

$$
\rho=1 ; \quad u=[1.25,1.25] ; \quad T=0.16 .
$$

Starting from a bimodal distribution one would expect the solution to reach the equilibrium state which is nothing else but a Maxwellian distribution with the same macroscopic quantities of the starting distribution $f$.

In Fig. 1, we reported the level set values of the distribution function $f$ for different times. We observe that the two initial Maxwellian distributions centered at different locations in the velocity space gradually merge into one. Once that the equilibrium 



FIGURE 1. Time evolution of the level set of distribution function $f$, starting from the double peak distribution (4.2). A first order exponential Runge-Kutta method is used for the time integration.

state is reached, the numerical scheme is able to preserve this steady state for all times. On the contrary, as already known, a standard spectral scheme is not able to preserve the steady state for infinite times since conservation of energy is, in general, not guarantee for this kind of schemes. In this test, the first-order exponential RungeKutta method is used. All other time integrators methods give analogous results, i.e. 

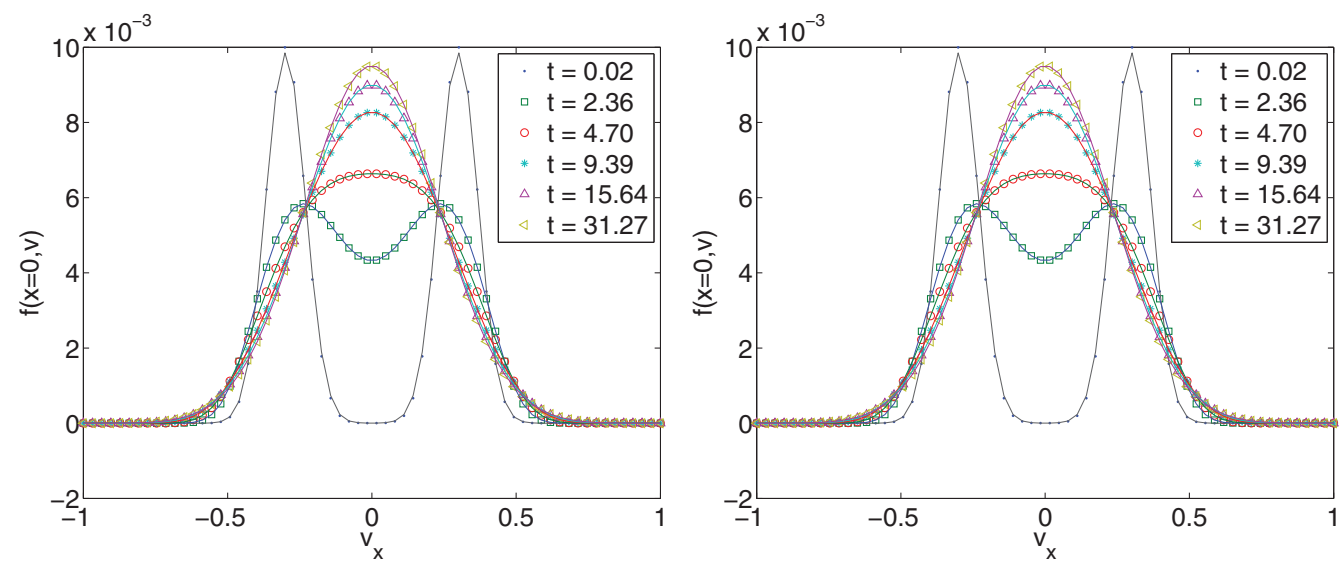

FIGURE 2. Time evolution of distribution function $f\left(v_{x}, v_{y}=0\right)$ at various time slot for Rosenbluth's test problem. Solid lines: reference solution computed by an explicit RK3 method with $\Delta t=1.0078 \times 10^{-4}$. Markers: solution computed by exponential RK3 method (left) and third order IMEX-RK method (right) with $\Delta t=0.0156$.

the steady state solution is reached and then preserved for all times and thus results for these schemes are not reported.

\subsubsection{Test 2: Rosenbluth's test.}

We perform a second simulation with an axially symmetric initial condition originated by the following distribution

$$
f(v, t=0)=0.01 \exp \left(-10\left(\frac{|v|-0.3}{0.3}\right)\right) .
$$

Scope of this simulation is to test the performances of the penalized time integrators schemes in terms of numerical errors in comparison to classical time integrators which should employ time steps restrictions for stability to be guaranteed.

In Fig. 2, we report the time evolution of the distribution function $f(v, t)$ for $v_{y}=0$. In this case, for the time integration, we employ the exponential RK3 method (left) and the third-order IMEX-RK method (right). Both schemes are compared with a reference solution computed by an explicit third-order Runge-Kutta scheme (solid line in both figures) which needs the stability condition $\Delta t \sim \Delta v^{2}$ to be satisfied. For the two schemes proposed, we use a time step $\Delta t=0.0156$ while to compute the reference solution, a time step is $\Delta t=1.0078 \times 10^{-4}$ is employed. The time step chosen for the penalized ERK and IMEX-RK schemes is chosen only in order to have sufficent precision in the solution and not for stability to be guaranteed. The figures show that even if much larger time steps are used for our methods, around 100 times bigger, the two penalized schemes and the reference solutions still match very well.

\subsubsection{Test 3: convergence rate test.}

Scope of this last space homogeneous test is to prove that the numerical convergence rates both for the exponential Runge-Kutta methods and for the IMEX-RK methods are close to the theoretical rates. The initial distribution is given by a double peak Gaussian (4.2) as in the previous test where now the corresponding macroscopic quantities are

$$
\rho=1, \quad u=[0.4,0], \quad T=0.8 .
$$




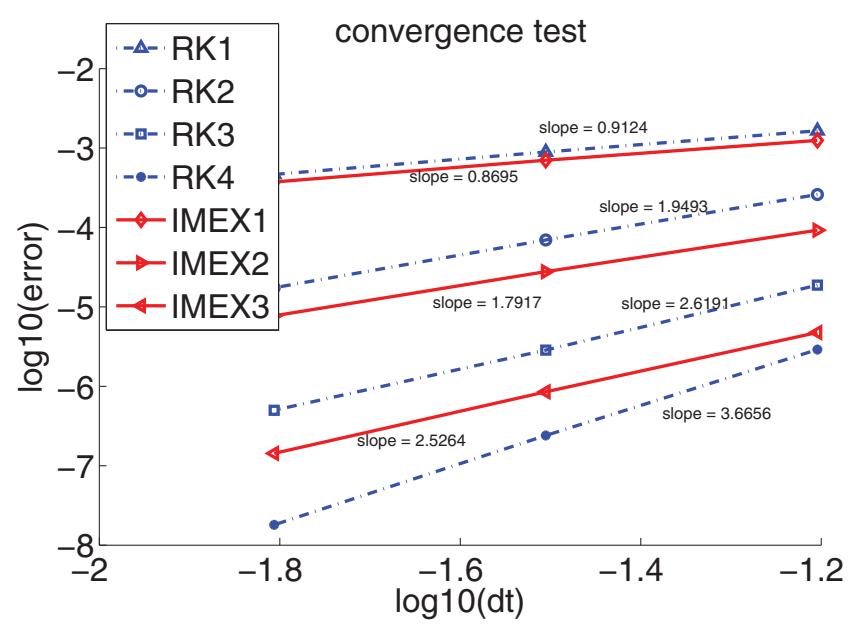

FIGURE 3. The convergence rates of the exponential Runge-Kutta methods (dotted line with markers) and of the IMEX-RK method (solid line with markers) for the homogeneous LFP equation with initial data (4.2) and (4.4).

We compute the $l^{1}$ error in time, fixing the final time at $t=0.5$, by

$$
\text { error }_{i}=\max _{v} \frac{\left\|f_{i}(t)-f_{i-1}(t)\right\|_{1}}{\left\|f_{i-1}(t)\right\|_{1}}
$$

and we measure its decay rate with respect to decreasing time steps $\Delta t$ keeping fixed the mesh in $v$. The time steps considered are respectively $\Delta t=1 / 16,1 / 32,1 / 64,1 / 128$ and for each value of the time step $\Delta t_{i}$ we compute the error, error ${ }_{i}$, by using the solution computed for $\Delta t_{i+1}$ as a reference solution.

The error curve expected are the ones corresponding to the time integrator used. This means we expect error curves with slope corresponding to respectively order one, two and three for the IMEX-RK schemes and up to order four for the ERK schemes since the discretization in velocity space is done by a spectral method and thus the error decay rate being much larger than the theoretical error decay rates expected by the time discretization schemes. In Fig. 3, the exponential Runge-Kutta methods (dotted line with markers) up to the fourth order and the IMEX-RK method (solid line with markers) up to the third-order are reported. The numerical decay rates obtained are within the expectation.

\subsection{The full non-homogeneous problem}

In this subsection, we analyze the behaviors of our numerical scheme on three different non-homogeneous test cases. We first consider a one dimensional in space and two dimensional in velocity space linear Landau damping and successively a nonlinear Landau damping, both problems are studied under different collisional regimes. First, we report the results in the non-collisional case and then we show what changes when collisions are added to the system. We report results for an average collision regime and a strong collisional regime. We successively consider a two stream instability problem again in the one in space-two in velocity space setting testing three different regimes : non-collisional, mild collisional and strong collisional.

For all tests the left-hand side of the collisional Vlasov equation is solved by a semiLagrangian method which employs cubic spline reconstructions of the distribution function and a fourth order splitting while the collisional part is solved by spectral 
methods in velocity and exponential integrators in time. The Poisson equation is solved by a fourth order finite difference method. The time step is fixed for all tests as $\Delta t \leqslant \min \left(\Delta x / v_{\max }, \Delta v / \max (|E|)\right.$. Let us recall that, due to the fact that semiLagrangian schemes are used for the transport terms, in principle a CFL condition is not needed, being the scheme unconditionally stable, consequently the above choice has been dictated solely by accuracy reasons. Let also observe that if standard explicit integrators instead of the uniformly stable integrators wants to be used, the time step should be fixed to $\Delta t \leqslant \varepsilon(\Delta v)^{2}$ which turns to be a strongly restrictive condition in practical situations.

\subsubsection{Test 1: linear Landau damping.}

The Vlasov-Poisson system is initialized by

$$
f\left(x, v_{x}, v_{y}\right)=\frac{1}{2 \pi}(1+\alpha \sin (k x)) e^{\frac{-v_{x}^{2}-v_{y}^{2}}{2}}, \quad\left(v_{x}, v_{y}\right) \in\left[-L_{v}, L_{v}\right]^{2}
$$

with $\alpha=0.01, k=1$ and a domain size $L=\frac{2 \pi}{k}$ with $L_{v}=6$. We run the test up to $T=10$ with three different Knudsen numbers:

(1) $\varepsilon=\infty$. The situation corresponds to solve the Vlasov equation and we expect the classical Landau damping behaviors.

(2) $\varepsilon=1$. The situation corresponds to mild collisions.

(3) $\varepsilon=10^{-4}$. The situation corresponds to very strong collisions. The distribution function is close to the Maxwellian state. In this regime, the Vlasov-Landau equation gives solutions very close to the one furnished by the corresponding fluid model.

The mesh is defined by $N=128$ and $N_{v}=128^{2}$ points for $\varepsilon=\infty$; while in the other two cases $N=64$ and $N_{v}=64^{2}$ is used since the collision term smooths the distribution function in velocity space and $N_{v}=64^{2}$ points are sufficient to get the analogous resolution of the non-collisional case. Periodic boundary conditions are used for the distribution function in space while in velocity space the distribution is set to $f=0$ at the boundaries. Finally, the Poisson equation is solved by employing Dirichlet boundary conditions on a staggered grid with respect to the one employed for solving the Vlasov equation. In Fig. 4, on the left we report the $L_{2}$ norm of the electric field versus time in logarithmic scale. The straight line represents the theoretical damping rate $\gamma=-0.85$. As expected in the collisionless case the damping computed is the same as the theoretical one while when collisions grows the damping is balanced by the effect of the interactions. In the limit of infinite collisions, the energy associated with the electric field oscillates around a constant value.

\subsubsection{Test 2: nonlinear Landau damping.}

In this case, the Vlasov-Poisson equation is initialized by

$$
f\left(x, v_{x}, v_{y}\right)=\frac{1}{2 \pi}(1+\alpha \cos (k x)) e^{\frac{-v_{x}^{2}-v_{y}^{2}}{2}}, \quad\left(v_{x}, v_{y}\right) \in \in\left[-L_{v}, L_{v}\right]^{2}
$$

with $\alpha=0.5, k=0.5$, and a domain size $L=\frac{2 \pi}{k}$ with $L_{v}=6$. We run the test up to $T=40$ with $N=128, N_{v}=128^{2}$ for the non-collisional case, while in the collisional case we used $N_{v}=64^{2}$, since as in the previous case, collisions smooth the distribution function in the velocity space and $N_{v}=64^{2}$ points turn out to be enough to reach the same precision of the non-collisional regime. We simulate this problem using three different Knudsen numbers:

(1) $\varepsilon=\infty$. The situation corresponds to solve the Vlasov equation and we expect as in the previous case the classical Landau damping behaviors. 



FIGURE 4. The linear Landau damping on the left and the nonlinear Landau damping on the right associated to different Knudsen numbers. The three figures, from top to bottom are for $\varepsilon=\infty, 1$ and $10^{-4}$, respectively.

(2) $\varepsilon=1$. The situation corresponds to mild collisions.

(3) $\varepsilon=10^{-4}$. The situation corresponds to very strong collisions. The distribution function is close to the Maxwellian state. In this regime, the Vlasov-Landau equation gives solutions very close to the one furnished by the corresponding fluid model.

The boundary conditions both for the distribution function and the Poisson equation are the same as in the previous test. In Fig. 4, on the right, we report the $L_{2}$ norm of the electric field versus time in logarithmic scale. In this situation, 
in the non-collisional case, the electric energy is first exponentially decreasing with a decay rate of approximately $\gamma_{1}=-0.2812$ as reported in the paper of Cheng and Knorr (1976) and Guo and Qiu (2013). After the decreasing phase, the growth rate phase is approximately defined by a growing rate $\gamma_{2}=0.08$ which is consistent with the results of Guo and Qiu (2013) and Heath et al. (2012). At variance, in presence of mild collisions, the electric energy is exponentially decreasing during all the simulation. In the strong collisional regime the behavior is opposite, the electric energy oscillates around a constant value as in the linear case.

\subsubsection{Test 3: two stream instability.}

In this case, the initial data are

$$
f\left(x, v_{x}, v_{y}\right)=\frac{1}{12 \pi}(1+\alpha \cos (k x)) e^{-\left(v_{x}^{2}+v_{y}^{2}\right) / 2}\left(1+5 v_{x}^{2}\right), \quad\left(v_{x}, v_{y}\right) \in\left[-L_{v}, L_{v}\right]^{2}
$$

with $\alpha=0.05, k=0.5$ and the domain size $L=[0,2 \pi / k]$ in the physical space and with $L_{v}=6$ in the velocity space. We run the test up to $T=40$ with three different Knudsen numbers:

(1) $\varepsilon=\infty$.

(2) $\varepsilon=1$.

(3) $\varepsilon=10^{-4}$.

The above situations correspond as before to three different collisional regimes. The boundary conditions are still periodic in space for the distribution $f$ and Dirichlet in velocity space. The Poisson equation employs Dirichlet conditions. The number of points is $N_{x}=128$ and $N_{v}=128^{2}$ for the non-collisional case while we choose $N_{v}=64^{2}$ in the mild and strong collisional cases. In Figs 5-7 we report on the top the isosurfaces while on the bottom the projections in the $x-v_{x}$ plane of the distribution $f$ for different times and different Knudsen numbers. In the collisionless case (Fig. 5), the formation of instability is observed and the results are consistent with the ones observed in Crouseilles et al. (2008). In the case of mild collisions (Fig. 6), we observe for $t=2$ the formation of a small hole in the center which is still present at $t=4$ even if it is very close to disappearance, then for $t=8$ the effect of the collisions become predominant and the distribution $f$ approaches rapidly the Maxwellian state. With strong collision (Fig. 7) the distribution function approaches the Maxwellian at a much larger rate and we only observe some oscillations of the distribution around the axis $v_{x}=0$ for $t=2$ and $t=4$.

\subsection{Efficiency test}

We finally perform some efficiency test to measure the performances of the scheme proposed. More in details, we measure the efficiency of the fast spectral method in comparison with the standard spectral method and we measure the computational cost of solving a collision term in comparison with the computational cost of solving only the collisionless Vlasov equation. We remind to a future work an analogous analysis where the effect of different time integrators is tested in term of computational costs.

\subsubsection{Test 1: fast spectral methods vs standard spectral methods.}

The theoretical computational cost of the fast spectral method described in Sec. 2.2.2 for evaluating the Landau operator $Q(f)$ is $n \log n$ where $n=N_{v}^{2}$ is the total number of grid points in velocity space. This fast summation method is compared to a standard spectral method, the one described in equation (2.10) with a theoretical cost of the order $n^{2}$ for a single evaluation of the same operator. 



FIGURE 5. Two stream instability without collision at time $t=1,10,20,40$. Top: 3D illustration; Bottom: projection in the $x-v_{x}$ plane. 



$t=1$
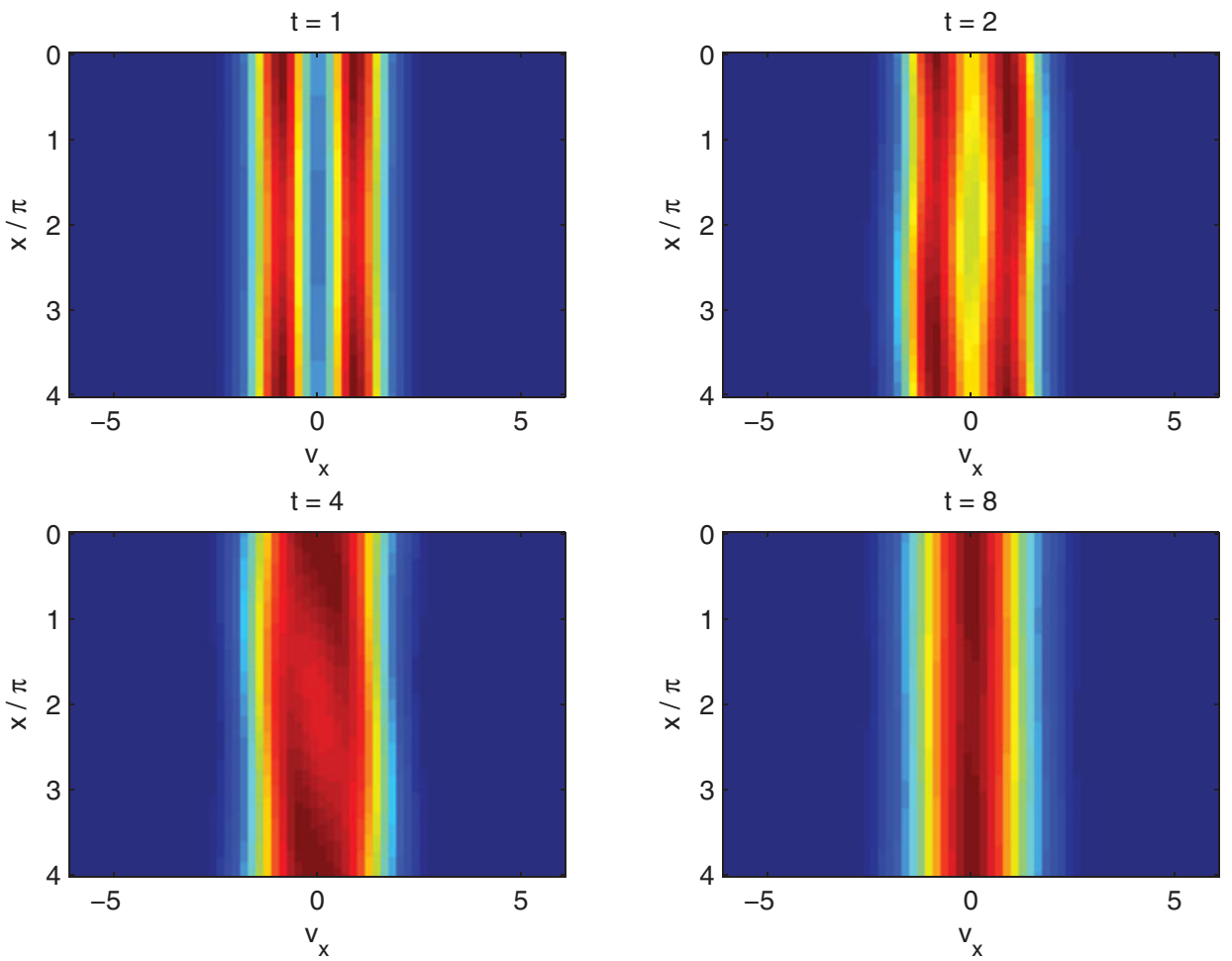

FIGURE 6. Two stream instability associated with Knudsen number $\varepsilon=1$ at time $t=1,2,4,8$. Top: 3D illustration; Bottom: projection in the $x-v_{x}$ plane. 



Figure 7. Two stream instability associated with Knudsen number $\varepsilon=10^{-4}$ at time $t=1,2,4,8$. Top: 3D illustration; Bottom: projection in the $x-v_{x}$ plane. 


$\begin{array}{lrrr} & N_{v}=32^{2} & N_{v}=64^{2} & N_{v}=128^{2} \\ \text { Fast spectral method } & 0.184 & 0.756 & 3.460 \\ \text { Standard spectral method } & 29.924 & 547.824 & 8891.244\end{array}$

TABLE 1. The comparison of the computation times (in seconds) between a standard spectral method and a fast spectral method.

$\begin{array}{lrrrc} & N_{v}=32^{2} & N_{v}=64^{2} & N_{v}=128^{2} & N_{v}=256^{2} \\ \text { 1 Vlasov solver } & 66.83 \% & 65.28 \% & 52.00 \% & 29.59 \% \\ \text { Evaluating } Q & 24.19 \% & 21.11 \% & 19.07 \% & 18.59 \% \\ \text { Inverting } P & 8.97 \% & 13.61 \% & 28.93 \% & 51.83 \%\end{array}$

TABLE 2. Relative computational times (in percentage) for the different parts.

In order to numerically test the two spectral approaches, we apply an explicit Euler method for integrating the Vlasov-Landau equation on a space homogeneous problem with a double peak Gaussian initial distribution as the one described in (4.2). The number of time steps is fixed to 100 and the total computation time cost is summarized in Table 1. The results show that the gain in term of computational cost is even larger than the theoretical one, however one has to observe that while there exists optimized routines for computing the fast Fourier transform, the summation which is needed for solving the problem by the classical spectral method is obtained with standard techniques.

\subsubsection{Test 2: transport vs collisions.}

We finally measure the computational times corresponding to the numerical solution of the collisionless Vlasov equation and of the homogeneous Landau equations when they are coupled together by means of splitting methods. In the following test, a third-order semi-Lagrangian method is applied to the Vlasov equation (2.2) while a fourth order exponential Runge-Kutta method is applied on the Landau collision part (2.1). A fourth-order time splitting (2.6) is applied to combine the two terms. We compare the CPU time spent on the computation of the Vlasov equation by the semi-Lagrangian method, the CPU time for the evaluation of the Landau operator $Q$ by the fast spectral method and the CPU time needed for inverting the linear Fokker-Planck operator $P$ by a conjugate gradient method. The initial data are those of the two stream instability problem (4.8) introduced before. The results are summarized in Table 2 for an increasing number of points $N_{v}$ in velocity space, the mesh in the physical space being fixed to $N=64$.

\section{Conclusions}

In the present paper, we considered the development of efficient and accurate numerical methods for the solution of the Vlasov-Landau equation describing a collisional plasma. The main advantage of the approach here presented is that it permits to embed easily and existing collisionless code with a collisional Landau code based on fast spectral methods and suitable high-order time integrators. If compared with existing deterministic schemes for collisional plasma, like the one derived in Filbet and Pareschi (2002), the method here presented has the following advantages. 
(a) The spectral approximation of the collision term is spectrally accurate, can be evaluated with fast algorithms, and captures correctly the Maxwellian steady states;

(b) The time discretization of the collisional phase can achieve high-order accuracy and is capable to avoid the parabolic stiffness induced by the Landau operator;

(c) Close to thermodynamic equilibrium, or alternatively in the limit of vanishing Knudsen number, the numerical method is consistent with the Euler-Poisson fluid approximation of the plasma.

\section{REFERENCES}

Alexandre, R. and Villani, C. 2004 On the Landau approximation in plasma physics. Ann. Inst. Henri Poincaré Anal. Non Linéaire 21(1), 61-95.

Arsenév, A. A. 1989 On a connection between the Boltzmann equation and the Landau-FokkerPlanck equations. Dokl. Akad. Nauk SSSR 305(2), 322-324.

Ascher, U. M., Ruuth, S. J. and Spiteri, R. J. 1997 Implicit-explicit runge-kutta methods for time-dependent partial differential equations. Appl. Numer. Math. 25, 151-167.

Ayuso, B., Carrillo, J. A. and Shu, C.-W. 2011 Discontinuous Galerkin methods for the onedimensional Vlasov-Poisson system. Kinet. Relat. Models 4(4), 955-989.

Bobylev, A. and Rjasanow, S. 1997 Difference scheme for the Boltzmann equation based on the fast Fourier transform. Eur. J. Mech. B Fluids 16(2), 293-306.

Bobylev, A. V. and Nanbu, K 2000 Theory of collision algorithms for gases and plasmas based on the boltzmann equation and the landau-fokker-planck equation. Phys. Rev. E 61, 4576-4586.

Bobylev, A. V., Potapenko, I. F. and Chuyanov, V. A. 1980 Completely conservative difference schemes for nonlinear kinetic equations of Landau (Fokker-Planck) type. Akad. Nauk SSSR Inst. Prikl. Mat. Preprint 26.

Bobylev, A. V. and Rjasanow, S. 1999 Fast deterministic method of solving the Boltzmann equation for hard spheres. Eur. J. Mech. B Fluids 18(5), 869-887.

Boris, J. P. and Book, D. L. 1973 Flux-corrected transport I. SHASTA, a fluid transport algorithm that works. J. Comput. Phys. 11, 38-69.

Buet, C. and Cordier, S. 1998 Conservative and entropy decaying numerical scheme for the isotropic Fokker-Planck-Landau equation. J. Comput. Phys. 145(1), 228-245.

Buet, C. and Cordier, S. 1999 Numerical analysis of conservative and entropy schemes for the Fokker-Planck-Landau equation. SIAM J. Numer. Anal. 36(3), 953-973 (electronic).

Buet, C., Cordier, S., Degond, P. and Lemou, M. 1997 Fast algorithms for numerical, conservative, and entropy approximations of the Fokker-Planck-Landau equation. J. Comput. Phys. 133(2), $310-322$.

Buet, C., Cordier, S. and Filbet, F. 1999 Comparison of numerical schemes for Fokker-PlanckLandau equation. In: CEMRACS 1999 (Orsay), Proc. ESAIM, Vol. 10, Soc. Math. Appl. Indust., Paris, pp. 161-181 (electronic).

Caflisch, R., Jin, S. and Russo, G. 1997 Uniformly accurate schemes for hyperbolic systems with relaxation. SIAM J. Numer. Anal. 34(1), 246-281.

Caflisch, R., Wang, C., Dimarco, G., Cohen, B. and Dimits, A. 2008 A hybrid method for accelerated simulation of Coulomb collisions in a plasma. Multiscale Model. Simul. 7(2), 865-887.

Canuto, C., Hussaini, M. Y., Quarteroni, A. and Zang, T. A. 1988 Spectral Methods in Fluid Dynamics, New York: Springer-Verlag.

Carrillo, J. A. and Vecil, F. 2007 Nonoscillatory interpolation methods applied to Vlasov-based models. SIAM J. Sci. Comput. 29, 11791206.

Cercignani, C. 1988 The Boltzmann Equation and its Applications (Applied Mathematical Sciences, 67). New York: Springer-Verlag.

Cercignani, C., Illner, R. and Pulvirenti, M. 1994 The Mathematical Theory of Dilute Gases (Applied Mathematical Sciences, 106). New York: Springer-Verlag.

Cheng, C. Z. and Knorr, G. 1976 The integration of the Vlasov equation in configuration space. Comput. Phys. Commun. 22, 330-335.

Cheng, Y., Gamba, I. M. and Proft, J. 2012 Positivity-preserving discontinuous Galerkin schemes for linear Vlasov-Boltzmann transport equations. Math. Comput. 81(277), 153-190. 
Crouseilles, N., Gutnic, M., Latu, G. and Sonnendrücker, E. 2008 Comparison of two Eulerian solvers for the four-dimensional Vlasov equation. I. Commun. Nonlinear Sci. Numer. Simul. 13(1), 88-93.

Crouseilles, N., Mehrenberger, M. and Sonnendrücker, E. 2010 Conservative semi-lagrangian schemes for Vlasov-type equations. J. Comput. Phys. 229, 1927-1953.

Degond, P. 2014 Asymptotic-preserving schemes for fluid models of plasmas. Panoramas et Syntheses.

Degond, P. and Lucquin-Desreux, B. 1992 The Fokker-Planck asymptotics of the Boltzmann collision operator in the Coulomb case. Math. Models Methods Appl. Sci. 2(2), 167-182.

Degond, P. and Lucquin-Desreux, B. 1994 An entropy scheme for the Fokker-Planck collision operator of plasma kinetic theory. Numer. Math. 68(2), 239-262.

Desvillettes, L. 1992 On asymptotics of the Boltzmann equation when the collisions become grazing. Transp. Theory Stat. Phys. 21(3), 259-276.

Dia, B. O. and Schatzman, M. 1996 Commutateurs de certains semi-groupes holomorphes et applications aux directions alternées. RAIRO Modél. Math. Anal. Numér. 30(3), 343-383.

Dimarco, G., Caflisch, R. and Pareschi, L. 2010 Direct simulation Monte Carlo schemes for Coulomb interactions in plasmas. Commun. Appl. Ind. Math. 1(1), 72-91.

Dimarco, G. and Pareschi, L. 2011 Exponential Runge-Kutta methods for stiff kinetic equations. SIAM J. Numer. Anal. 49(5), 2057-2077.

Dimarco, G. and Pareschi, L. 2012 High order asymptotic-preserving schemes for the Boltzmann equation. C. R. Math. 350(910), 481-486.

Dimarco, G. and Pareschi, L. 2013 Asymptotic preserving implicit-Explicit Runge-Kutta methods for nonlinear kinetic equations. SIAM J.Numer. Anal. 51(2), 1064-1087.

Dimarco, G. and Pareschi, L. 2014 Numerical methods for kinetic equations. Acta Numer. 23, 369-520.

Filbet, F. and Jin, S. 2010 A class of asymptotic-preserving schemes for kinetic equations and related problems with stiff sources. J. Comput. Phys. 229(20), 7625-7648.

Filbet, F. and Mouhot, C. 2011 Analysis of spectral methods for the homogeneous Boltzmann equation. Trans. Am. Math. Soc. 363(4), 1947-1980.

Filbet, F., Mouhot, C. and Pareschi, L. 2006 Solving the Boltzmann equation in $N \log _{2} N$. SIAM J. Sci. Comput. 28(3), 1029-1053 (electronic).

Filbet, F. and Pareschi, L. 2002 A numerical method for the accurate solution of the Fokker-PlanckLandau equation in the nonhomogeneous case. J. Comput. Phys. 179(1), 1-26.

Filbet, F. and Pareschi, L. 2003 Numerical solution of the Fokker-Planck-Landau equation by spectral methods. Commun. Math. Sci. 1(1), 206-207.

Filbet, F., Pareschi, L. and Rey, T. 2014 On steady state preserving spectral methods for homogeneous Boltzmann equations. C. R. Acad. Sci. (submitted).

Filbet, F., Sonnendrücker, E. and Bertrand, P. 2001 Conservative numerical schemes for the Vlasov equation. J. Comput. Phys. 172(1), 166-187.

Gamba, I. M. and Haack, J. R. 2014 A conservative spectral method for the Boltzmann equation with anisotropic scattering and the grazing collisions limit. J. Comput. Phys. 270, 40-57.

Gamba, I. M. and Tharkabhushanam, S. H. 2009 Spectral-Lagrangian methods for collisional models of non-equilibrium statistical states. J. Comput. Phys. 228(6), 2012-2036.

Ghanshyam, N. and Tripathi, V. K. 1993 Self-focusing and filamentation of laser beams in collisional plasmas with finite thermal conduction. J. Plasma Phys. 49, 243-253.

Guo, W. and Qiu, J.-M. 2013 Hybrid semi-Lagrangian finite element-finite difference methods for the Vlasov equation. J. Comput. Phys. 234, 108-132.

Hairer, E., Lubich, C. and Wanner, G. 2010 Geometric Numerical Integration (Springer Series in Computational Mathematics, 31). Springer, Heidelberg, structure-preserving algorithms for ordinary differential equations, Reprint of the second (2006) edition.

Heath, R. E., Gamba, I. M., Morrison, P. J. and Michler, C. 2012 A discontinuous Galerkin method for the Vlasov-Poisson system. J. Comput. Phys. 231(4), 1140-1174.

Hochbruck, M. and Ostermann, A. 2010 Exponential integrators. Acta Numer. 19, 209-286.

Hu, J., Li, Q. and Pareschi, L 2014 Asymptotic-preserving exponential methods for the quantum Boltzmann equation with high-order accuracy. J. Sci. Comput. (to appear).

Jin, S. 1995 Runge-Kutta methods for hyperbolic conservation laws with stiff relaxation terms. $J$. Comput. Phys. 122(1), 51-67. 
Jin, S. 1999 Efficient asymptotic-preserving (ap) schemes for some multiscale kinetic equations. SIAM J. Sci. Comput. 21, 441-454.

Jin, S 2012 Asymptotic preserving (ap) schemes for multiscale kinetic and hyperbolic equations: a review. Riv. Mat. Univ. Parma 3 177-216.

Jin, S. and Yan, B. 2011 A class of asymptotic-preserving schemes for the Fokker-Planck-Landau equation. J. Comput. Phys. 230(17), 6420-6437.

Khabibrakhmanov, I. K. and Khazanov, G. V. 2000 The spectral collocation method for the kinetic equation with the nonlinear two-dimensional coulomb collisional operator. J. Comput. Phys. 161, 558-575.

Landau, L. D. 1936 Die kinetische gleichung für den fall coulombscher vechselwirkung (the transport equation in the case of the coulomb interaction). Phys. Z. Sowjet. 154.

Lemou, M. 1998 Multipole expansions for the Fokker-Planck-Landau operator. Numer. Math. 78(4), 597-618.

Li, Q. and Pareschi, L. 2014 Exponential Runge-Kutta for the inhomogeneous Boltzmann equations with high order of accuracy. J. Comput. Phys. 259, 402-420.

Li, Q., Pareschi, L. and Yan, B. 2014 Efficient time integration of the Fokker-Planck-Landau equation. preprint.

Li, Q. and Yang, X. 2014 Exponential Runge-Kutta methods for the multi-species Boltzmann equation. Commun. Comput. Phys. 15, 996-1011.

Moler, C. and Loan, C. V. 1978 Nineteen dubious ways to compute the exponential of a matrix. SIAM Rev. 20, 801-836.

Mouhot, C. and Pareschi, L. 2004 Fast methods for the Boltzmann collision integral. C. R. Math. Acad. Sci. Paris 339(1), 71-76.

Mouhot, C. and Pareschi, L. 2006 Fast algorithms for computing the Boltzmann collision operator. Math. Comput. 75(256), 1833-1852 (electronic).

Pareschi, L. and Perthame, B. 1996 A Fourier spectral method for homogeneous Boltzmann equations. In: Proc. 2nd Int. Workshop on Nonlinear Kinetic Theories and Mathematical Aspects of Hyperbolic Systems (Sanremo, 1994), Vol. 25, pp. 369-382.

Pareschi, L. and Russo, G. 1999 An introduction to Monte Carlo methods for the Boltzmann equation. In: CEMRACS 1999 (Orsay), Proc. ESAIM, Vol. 10, Soc. Math. Appl. Indust., Paris, pp. 35-76.

Pareschi, L. and Russo, G. 2000a Numerical solution of the Boltzmann equation. I. Spectrally accurate approximation of the collision operator. SIAM J. Numer. Anal. 37(4), 1217-1245.

Pareschi, L. and Russo, G. 2000b On the stability of spectral methods for the homogeneous Boltzmann equation. In: Proc. 5th Int. Workshop on Mathematical Aspects of Fluid and Plasma Dynamics (Maui, HI, 1998), Vol. 29, pp. 431-447.

Pareschi, L. and Russo, G. 2005 Implicit-Explicit Runge-Kutta schemes and applications to hyperbolic systems with relaxation. J. Sci. Comput. 25(1-2).

Pareschi, L. and Russo, G. 2011 Efficient asymptotic preserving deterministic methods for the boltzmann equation. In: Models and Computational Methods for Rarefied Flows, AVT- 194 RTO AVT/VKI. Rhode St. Genese, Belgium.

Pareschi, L., Russo, G. and Toscani, G. 2000a Fast spectral methods for the Fokker-Planck-Landau collision operator. J. Comput. Phys. 165(1), 216-236.

Pareschi, L., Russo, G. and Toscani, G. 2000b Méthode spectrale rapide pour l'équation de FokkerPlanck-Landau. C. R. Acad. Sci. Paris Sér. I Math. 330(6), 517-522.

Pareschi, L., Toscani, G. and Villani, C. 2003 Spectral methods for the non cut-off Boltzmann equation and numerical grazing collision limit. Numer. Math. 93(3), 527-548.

Pitale, L. A. 1978 Filamentation of a laser beam in a strongly ionized magnetoplasma. J. Plasma Phys. 19, 55-61.

Qiu, J.-M. and Shu, C.-W. 2011 Positivity preserving semi-lagrangian discontinuous Galerkin formulation: theoretical analysis and application to the VlasovPoisson system. J. Comput. Phys. 230, 8386-8409.

Rosenbluth, M. N., MacDonald, W. M. and Judd, D. L. 1957 Fokker-Planck equation for an inverse-square force. Phys. Rev. 107(2), 1-6.

Sonnendrücker, E. 2013 Numerical methods for Vlasov equations. Tech. Rep.. MPI TU Munich, (http://www-m16.ma.tum.de/foswiki/pub/M16/Allgemeines/NumMethVlasov/NumMeth-Vlasov-Notes.pdf). 
Sonnendrücker, E., Roche, J., Bertrand, P. and Ghizzo, A. 1999 The semi-Lagrangian method for the numerical resolution of the Vlasov equation. J. Comput. Phys. 149(2), 201-220.

Strang, G. 1968 On the construction and comparison of difference schemes. SIAM J. Numer. Anal. 5, 506-517.

Sydora, R. D., Detering, F., Rozmus, W., Bychenkov, Y. Yu., Brantov, A. and Capjack, C. E. 2006 Collisional particle simulation of ion acoustic instability. J. Plasma Phys. 72, 1295-1298.

Valentini, F., Onofri, M. and Primavera, L. 2009 Mixed finite difference-spectral numerical approach for kinetic and fluid description of nonlinear phenomena in plasma physics. In: Numerical Simulation Research Progress, New York: Nova Sci. Publ. pp. 99-139.

Valentini, F., Trávníček, P., Califano, F., Hellinger, P. and Mangeney, A. 2007 A hybrid-Vlasov model based on the current advance method for the simulation of collisionless magnetized plasma. J. Comput. Phys. 225(1), 753-770.

Villani, C. 2002 A review of mathematical topics in collisional kinetic theory. In: Handbook of Mathematical Fluid Dynamics, Vol. I, Amsterdam: North-Holland, pp. 71-305.

Wang, C., Lin, T., Caflisch, R., Cohen, B. I. and Dimits, A. M. 2008 Particle simulation of Coulomb collisions: comparing the methods of Takizuka \& Abe and Nanbu. J. Comput. Phys. 227(9), $4308-4329$. 